\title{
Effect of Remote and Local Acupuncture Points on Periarthritis of Shoulder: A Comparative Study
}

\author{
Kumaresan Poorna Chandran ${ }^{1, *}$, Prabu Poorna Chandran², Naveena Arumugam³ ${ }^{3}$ Sendhilkumar Muthappan ${ }^{4}$ \\ ${ }^{1}$ Department of Yoga, Government Yoga and Naturopathy Medical College \& Hospital, Arumbakkam, Chennai, India \\ ${ }^{2}$ Department of Acupuncture and Energy Medicine, Govt. Yoga and Naturopathy Medical College \& Hospital, Arumbakkam, Chennai, India \\ ${ }^{3}$ Department of Yoga and Naturopathy, Southern Railway Head Quarters Hospital, Perambur, Chennai, India \\ ${ }^{4}$ Independent Researcher, Chennai, India
}

Received October 16, 2019

Revised January 3, 2021

Accepted January 18, 2021

Correspondence to Kumaresan Poorna Chandran Department of Yoga, Government Yoga and Naturopathy Medical College \& Hospital, Arumbakkam, Chennai, India

E-mail dr.k.bnys@gmail.com
Background: Periarthritis of shoulder is a painful condition of the shoulder, affecting 2-3\% of the general population and $20 \%$ of diabetic patients. Acupuncture is a widely practiced traditional Chinese medicine. Recent evidence shows that it alleviates shoulder pain with different needling techniques.

Objectives: The present study is to compare the efficacy of remote and local points on PAS.

Methods: 60 subjects were randomly assigned into two groups, remote acupuncture group $(n=30)$ and local acupuncture group $(n=30)$. Both groups were assessed at baseline and at the end of 12 sessions. Shoulder pain and its disability index (SPADI) and (ROM) were measured using goniometer. The intervention was given weekly thrice on alternate days for four weeks with 20 min for each session.

Results: The result shows that both remote and local acupuncture points were beneficial in the pain management and rage of motion when compared within the group. The effects of acupuncture at remote acupoints were better than those at local acupoints in SPADI and ROM when compared between two groups.

Conclusion: In treatment of periarthritis of shoulder remote acupuncture points may have higher therapeutic value when compared to local points.

Keywords: Acupuncture, Frozen shoulder, Periarthritis of shoulder, Range of motion, Shoulder pain

\section{INTRODUCTION}

Periarthritis of shoulder (PAS) is a painful ailment of shoulder [1]. Neviaser [2] in 1945 coined the term 'adhesive capsulitis' for painful stiffens of the shoulder. Later Codman [3] used another term called 'Frozen shoulder' to describe insidious onset of pain felt near the insertion of the deltoid muscle, difficulty in sleeping on the affected side, restriction in both active and passive elevation and external rotation, without any radiological changes. PAS is a clinical diagnosis rule out from a history of the gradual onset of severe shoulder pain with the progressive limitation of both active and passive glenohumeral movements $[4,5]$. PAS has an incidence of 3\% to $5 \%$ in the general population and up $20 \%$ in those with diabetes [6]. This condition has commonly reported the burden of middle age between 40 years to 60 years and to some extent common in women [7].
Extended immobilization of a joint has been shown to cause several detrimental pathophysiologic findings including decreased collagen length, fibro-fatty infiltration into the capsular recess, ligament atrophy resulting in reduced stress absorption, collagen band bridging across recesses, random collagen production, and reformed sarcomere number in muscle tissue [8].

There are many intervention options available for PAS, such as anti-inflammatory agents, intra-articular corticosteroid injections [9], capsular distension injections [10], bupivacaine suprascapular nerve blocks [11], manipulation under anesthesia [12], arthroscopic release and repair [13]. Most of the patients may also choose complementary therapies like physiotherapy [14], exercise therapy, electrotherapy, hydrotherapy and acupuncture for the management of pain and to progress Range of motion (ROM) of the affected joint $[15,16]$.

Acupuncture is traditional Chinese medicine and it has 
been described as an effective treatment approach for various diseases [17]. Acupuncture involves the insertion of needles at specific points of the body, which has functional specificity [18]. In recent decades, acupuncture as a complementary and alternative therapy has been widely recognized in western countries. Current evidence suggests that acupuncture is an effective intervention for treating pain and musculoskeletal disorders [19].

Studies report that acupuncture is effective for PAS; however, the strength of evidence and its recommendation is still weak. According to a recent Cochrane systematic review, the effect of acupuncture for shoulder pain and its function improvement is still unconvincing [20]. Current diminutive evidence is unable to affirm the efficacy of acupuncture for PAS. It is of great importance to conduct a clinical trial with a rigorous methodology to assess the effectiveness of acupuncture.

Different acupuncture points possess different treatment effects. Our clinical observation indicates that the combined needling on remote and local acupuncture points may be effective in treating PAS and restores the function of the shoulder joint. But, at present, there is still no comparative study to evaluate the effect of remote and local points for PAS. Therefore, we carried out a randomized comparative trial to assess the independent effect of four local acupuncture points and four remote acupuncture points [21] for PAS on pain and range of motion in PAS.

\section{MATERIALS AND METHODS}

\section{Study setting}

Subjects were recruited from the out-patient Department of Government Yoga \& Naturopathy Medical College and Hospital, Chennai.

\section{Study period}

Study was conducted from August 2017 to November 2017.

\section{Study population}

The research population for this study comprised age ranging between 40-65 years with both gender. Subjects were recruited according to the inclusion and exclusion criteria. Subjects were included in the study after obtain of written informed consent.

\section{Inclusion criteria}

Shoulder pain for more than 1 month and less than 12-month duration, restriction of abduction, flexion and external rotation, pain at night with the inability to lie on the affected side, aged between 40 years and 65 years of both gender, not received acupuncture in the last 4 weeks for PAS.
Providing written informed consent and agree to participate were included in the study.

\section{Exclusion criteria}

Participants will be excluded if they have history of major shoulder injury or surgery, cervical radiculopathy, paresis or other neurological changes in the upper limb on the involved side, presence of underlying fracture associated inflammatory arthritis, known renal or hepatic disease, haematopoietic disorder, malignancy, psychological disorder or under any psychiatry drugs, uncontrolled diagnosed neurological diseases, immunodeficiency, bleeding disorders and allergies, women in lactation, pregnant women.

\section{Type of the design}

A randomized clinical trial with an active control group.

\section{Randomization}

A total of eighty eight samples were screened, out of which sixty subjects were met the eligibility criteria and they were randomly divided into remote acupuncture points group $(\mathrm{n}=30)$ and Local Acupuncture points Group ( $\mathrm{n}=$ 30). Randomization was generated by using the website randomization.com (http://www.randomization.com) (Fig. 1).

\section{Intervention}

Selected remote acupuncture points with their Chinese name, location and needling method are explained in the Table 1, similarly local acupuncture points are explained in Table 2.

\section{Assessment tools}

\section{1) Shoulder pain and disability index (SPADI)}

SPADI were used to measure present shoulder pain and its disability. It is a self-administered questionnaire that consists of 13 items to assess two domains, pain (5 items) and disability (8 items). The items of both domains were scored on a visual analog scale (VAS) ranging from 0 to 10 , where $0=$ no pain/no difficulty and $10=$ worst pain [22]. Subjects were assessed twice and the questionnaire was administered at the baseline and after completion of 12 sessions (Appendix 1) and total scores in pain and disability were analysed.

\section{2) Instructions for scoring}

The subject was instructed to mark a circle on the score for each item that best represented their experience of their shoulder condition [23]. Subscale was summed and percentage was calculated out of each subscale and also for the total score. Mean was taken of the two subscales to give a total score out of 10, a higher score indicating greater impairment 
Enrollment
Assessed for eligibility $(n=88)$

Excluded $(n=28)$

- Not meeting inclusion criteria $(n=20)$

- Declined to participate $(n=5)$

- Other reasons $(n=3)$

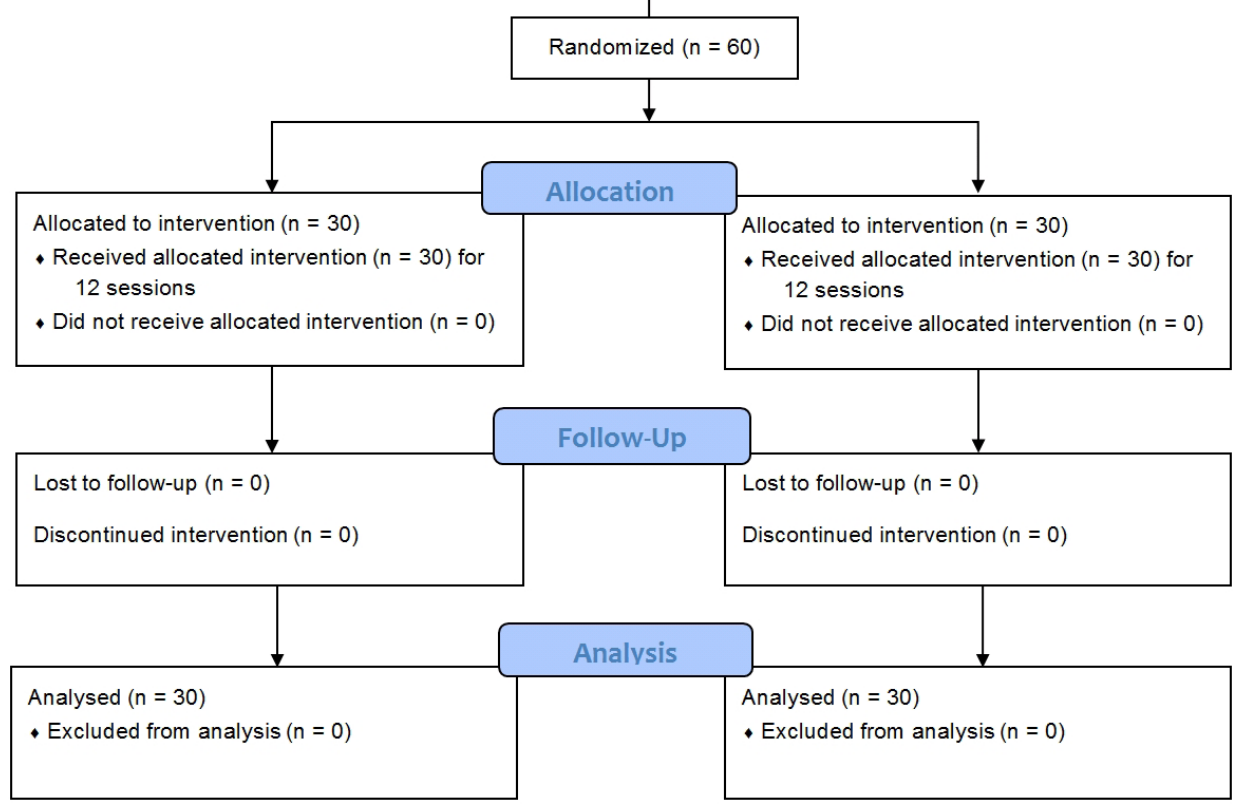

Fig. 1. Randomization of the study patients.

Table 1. Description and methods of remote acupuncture points

\begin{tabular}{|c|c|c|c|}
\hline $\begin{array}{l}\text { Acupuncture } \\
\text { points }\end{array}$ & Chinese name & Location & Needling method \\
\hline ST38 & Tiaokou & $\begin{array}{l}\text { One finger breath lateral to the inferior end of the tibial tuberosity is } \\
\text { ST36 (Zusanli). ST38 is located } 5 \text { cun below ST36 and one finger } \\
\text { breadth lateral to the anterior border of the tibia. }\end{array}$ & 1 cun perpendicular. \\
\hline GB34 & Yanglingquan & $\begin{array}{l}\text { Located on the antero-lateral aspect of the leg below the head of the } \\
\text { fibula. }\end{array}$ & 1 cun perpendicular. \\
\hline GB41 & Zulinqi & $\begin{array}{l}\text { Anterior depression between 4th and 5th metatarsal of the foot. This is } \\
\text { distal point for PAS. }\end{array}$ & 0.5 cun perpendicular. \\
\hline BL11 & Dazhu & $\begin{array}{l}\text { Located on the upper back, } 1.5 \text { cun lateral to the tip of the spinous } \\
\text { process of the } 1 \text { st thoracic vertebra. }\end{array}$ & 0.5 cun obliquely downwards. \\
\hline
\end{tabular}

$\mathrm{ST}=$ Stomach Meridian; GB = Gall Bladder Meridian; BL = Bladder Meridian .

Table 2. Description and methods of local acupuncture points

\begin{tabular}{|c|c|c|c|}
\hline $\begin{array}{l}\text { Acupuncture } \\
\text { points }\end{array}$ & Chinese name & Location & Needing method \\
\hline LI15 & Jianyu & $\begin{array}{l}\text { Located in between acromion process and greater tuberosity of the } \\
\text { humerus. When the arm is abducted two hollows appear on the shoulder. } \\
\text { The anterior hollow is LI15. }\end{array}$ & $\begin{array}{l}1 \text { cun, straight with upper arm } \\
\text { was positioned horizontal in } \\
\text { abduction. }\end{array}$ \\
\hline TE14 & Jianliao & $\begin{array}{l}\text { Located in between the acromion process and greater tuberosity of the } \\
\text { humerus. When the arm is abducted two hollows appear on the shoulder. } \\
\text { The posterior hollow is TE14. }\end{array}$ & $\begin{array}{l}1 \text { cun, straight with upper arm } \\
\text { was positioned horizontal in } \\
\text { abduction. }\end{array}$ \\
\hline GB21 & Jianjing & $\begin{array}{l}\text { Located at the highest point of the shoulder joint. Surface marking was } \\
\text { between the 7th intervertebral disc and acromion. }\end{array}$ & 0.5 cun, perpendicular. \\
\hline SI9 & Jianzhen & $\begin{array}{l}\text { Located } 1 \text { cun above the lower margin of the posterior axillary fold; arm } \\
\text { should be kept in full adduction. }\end{array}$ & 0.5 cun, straight. \\
\hline
\end{tabular}

$\mathrm{LI}$ = Large Intestine Meridian; TE = Triple Energizer Meridian; GB = Gall Bladder Meridian; SI = Small Intestine Meridian . 
or disability. In each subscale, patients are advised to mark only one item. If the item was not applicable to them, they are requested to pass to the next question. The percentage was calculated by excluding the unanswerable item from the total score. If a patient marks more than two items that item was considered as invalid entry and no score was given.

\section{3) Range of shoulder joint}

Ranges of motion (ROM) measurements are essential for the evaluation and diagnosis of PAS [24]. Goniometry is used to measure the range of motion as degree at a specific joint [25]. Active range of motion of flexion, abduction and external rotation was assessed at the neutral position.

\section{4) Acupuncture needles}

1 cun $(0.25 \times 0.25)$ sterile disposable needles were used. During each session needle was remains in the acupuncture point for $20 \mathrm{~min}$ without any stimulation.

\section{Data extraction}

The data was collected as primary outcomes (SPADI) and secondary outcome variables (ROM). The assessments were done on them before the day of intervention (baseline data) and after completion of 12 sessions of acupuncture intervention (post data).

\section{Data analysis}

Data were analyzed using IBM SPSS 18.0. The data were checked for normality by Shapiro-Wilk test. Statistical significance of differences between the values of SPADI and ROM was defined as a $p<0.05$ by the Student's t-test.

\section{Ethical consideration}

Ethical approval has been obtained from the Institutional Ethical Committee, Government Yoga and Naturopathy Medical College \& Hospital, Arumbakkam, Chennai.

\section{RESULTS}

We recruited 88 subjects. After exclusion of 28 subjects, 60 subjects were included for this study. The subjects were divided equally into 2 groups. The mean age of remote and local acupuncture group were 53.46 (SD 7.88) and 54.36 (SD 7.62) respectively (Table 3 ).

In both Remote and Local groups, the data were compared with pre and post-intervention, it showed that significant

Table 3. Demographic description of the study participants

\begin{tabular}{lcc}
\multicolumn{1}{c}{ Contents } & $\begin{array}{c}\text { Remote acupuncture } \\
\text { group (30) }\end{array}$ & $\begin{array}{c}\text { Local acupuncture } \\
\text { group (30) }\end{array}$ \\
\hline Age (Mean \pm SD) & $53.46 \pm 7.88$ & $54.36 \pm 7.62$ \\
Gender & $13(44 \%)$ & $15(50 \%)$ \\
Male & $17(56 \%)$ & $15(50 \%)$ \\
Female & & \\
\hline
\end{tabular}

$\mathrm{SD}=$ Standard deviation

Table 4. Shoulder pain and disability index before and after acupuncture

\begin{tabular}{|c|c|c|c|c|c|c|c|c|}
\hline \multirow{3}{*}{ Variables } & \multicolumn{4}{|c|}{ Remote acupuncture group } & \multicolumn{4}{|c|}{ Local acupuncture group } \\
\hline & \multicolumn{2}{|c|}{ Mean (SD) } & \multirow{2}{*}{$\begin{array}{c}\text { Mean } \\
\text { difference }\end{array}$} & \multirow{2}{*}{${ }^{*} p$} & \multicolumn{2}{|c|}{ Mean (SD) } & \multirow{2}{*}{$\begin{array}{c}\text { Mean } \\
\text { difference }\end{array}$} & \multirow{2}{*}{$* p$} \\
\hline & Pre & Post & & & Pre & Post & & \\
\hline Pain index & $84.3(6.5)$ & $56.6(48.8)$ & 27.7 & $<0.001$ & $79.6(10.9)$ & $61.5(12.4)$ & 18.1 & $<0.001$ \\
\hline Disability index & $82.7(5.9)$ & $54.2(15.8)$ & 28.5 & $<0.001$ & $81.4(7.9)$ & $61.6(12.5)$ & 19.8 & $<0.001$ \\
\hline Total SPADI score & $83.3(5.9)$ & $55.1(15.1)$ & 28.2 & $<0.001$ & $80.7(8.7)$ & $61.8(12.3)$ & 18.9 & $<0.001$ \\
\hline
\end{tabular}

$p=$ Probability; ${ }^{*}$ Comparing mean between pre and post for each intervention group, ${ }^{\sharp}$ Comparing mean difference between two-intervention groups.

Table 5. Range of shoulder joint movement before and after acupuncture

\begin{tabular}{|c|c|c|c|c|c|c|c|c|}
\hline \multirow{3}{*}{ Variables } & \multicolumn{4}{|c|}{ Remote acupuncture group } & \multicolumn{4}{|c|}{ Local acupuncture group } \\
\hline & \multicolumn{2}{|c|}{ Mean (SD) } & \multirow{2}{*}{$\begin{array}{c}\text { Mean } \\
\text { difference }^{\#}\end{array}$} & \multirow{2}{*}{${ }^{*} p$} & \multicolumn{2}{|c|}{ Mean } & \multirow{2}{*}{$\begin{array}{c}\text { Mean } \\
\text { difference }\end{array}$} & \multirow{2}{*}{${ }^{*} p$} \\
\hline & Pre & Post & & & Pre & Post & & \\
\hline Flexion & $81.4(5.2)$ & $116.4(18.1)$ & -35.0 & $<0.001$ & $80.8(5.6)$ & $104.7(15.5)$ & -23.9 & $<0.001$ \\
\hline Abduction & $82.4(4.8)$ & $120.1(19.9)$ & -37.7 & $<0.001$ & $78.7(5.6)$ & $103.0(16.5)$ & -24.3 & $<0.001$ \\
\hline External rotation & $26.4(2.5)$ & $42.6(4.7)$ & -16.2 & $<0.001$ & $25.3(4.5)$ & $38.9(6.4)$ & -13.6 & $<0.001$ \\
\hline
\end{tabular}

$p=$ Probability; ${ }^{*}$ Comparing mean between pre and post for each intervention group, ${ }^{*}$ Comparing mean difference between two-intervention groups. 


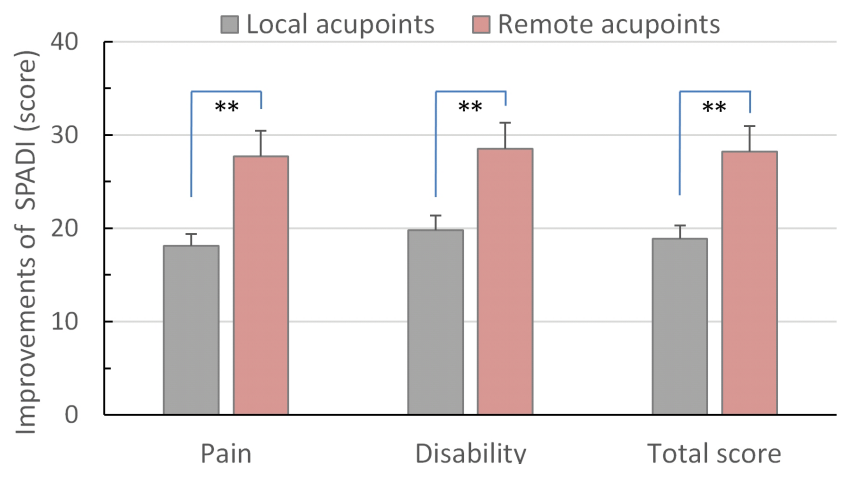

Fig. 2. Effects of acupuncture at local and remote acupoints on the shoulder pain and disability index. ${ }^{* *} p<0.01$ by student's t-test. SPADI, shoulder pain and disability index. Total score is the sum of pain and disability scores of individual patient.

improvement in the pain index, disability index, total SPADI score $(p<0.001$, Table 4 and 5$)$.

When the acupuncture-induced effects of Remote and Local acupuncture groups, the effects of acupuncture at remote acupoints on the shoulder pain and disability were significantly larger than those of acupuncture at local acupoints as illustrated in Fig. 2. Similarly, the effects of acupuncture at remote acupoints on the range of movement of shoulder joint were significantly larger than those at local acupoints as illustrated in Fig. 3. Taken together, we showed here that acupuncture at remote acupoints are more effective than that at local acupoints in the patients with periarthritis of shoulder although both remote and local acupoints stimulations were effective.

\section{DISCUSSION}

Acupuncture is a complementary and alternative therapy widely recognized in western countries [26]. Current evidence suggests that acupuncture is a valid intervention for treating pain and musculoskeletal disorders. In this study, we have selected remote acupuncture points and local acupuncture points as an intervention for PAS. SPADI questionnaire and ROM - flexion, abduction and external rotation are the variables used to measure the pain, disability, and restriction of shoulder joint.

This study revealed that both remote and local acupuncture stimulation alleviate the symptoms of periarthritis of shoulder in pain and disability index and also in the range of motion of flexion, abduction and external rotation. However remote acupuncture points ST38, GB34, GB41, BL11 shows the significant result when compared to that of local acupuncture points LI15, TE14, SI9, GB21 for 12 sessions.

A systemic review mentioned that there is [20] no adverse effect was observed during the during the 12 sessions with sixty subjects. A study by Chen [27] in 2006 shows that both

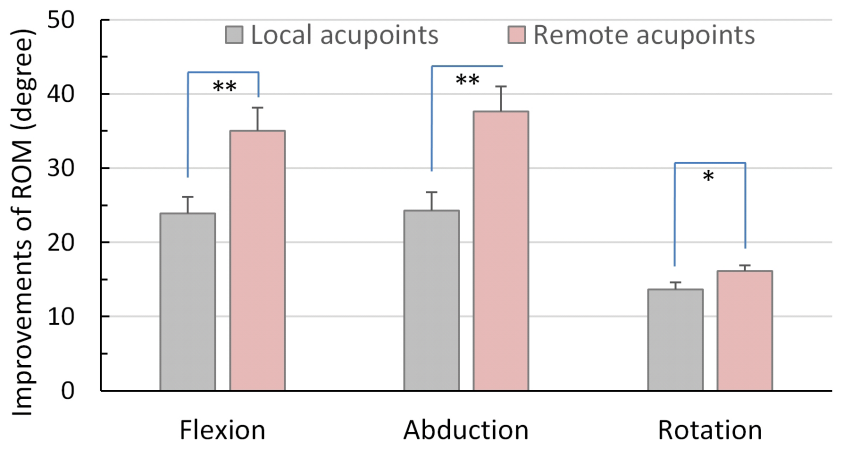

Fig. 3. Effects of acupuncture at local and remote acupoints on the range of motion (ROM). ${ }^{*} p<0.05$ and ${ }^{* *} p<0.01$ by student's t-test.

normal acupuncture and shallow needling with short needle was clinically benefited and there were no significant changes between the two groups. Whereas, in our present study we noticed that remote acupuncture stimulation was beneficial for the management of shoulder pain and disability than the local acupuncture group.

Few studies showed that three-phase acupuncture and fu's subcutaneous needle technique have significant therapeutic effect on PAS than the routine or local acupuncture stimulation $[28,29]$. Similarly, present study also showed acupuncture at remote acupoints has high therapeutic value in terms of both clinical and statistical when compared to local acupuncture.

The previous study on comparison between traditional, standard, sham acupuncture on SPADI showed that clinical improvements were noticed in traditional acupuncture points and standard acupuncture, our study results also showed that total SPADI score was improved in both remote and local acupuncture group. But remote acupuncture points were noticed marked improvement in the total SPADI score [30]. Another multi-centric study shows that acupuncture may help to alleviate the symptoms of PAS than the conservative orthopedic treatment [31]. Our study shows that symptoms of PAS like pain, restricted movements were improved after 12 sessions of acupuncture for 4 weeks.

Han et al. [32], in 2014 concluded that combined therapy of remote and local acupuncture stimulation with filiform and warm needling and positive functional exercise are beneficial for chronic stages of PAS. Whereas, in the present study showed remote acupuncture points alone have greater beneficial effect in shoulder ROM and to reduce pain without any combined therapy.

Another study has concluded that abdominal acupuncture show significant changes in both pain and adhesion stages of PAS [33]. In this study, we have included acute and chronic stages of PAS. We found that both acute and chronic stages of PAS could get improvement in ROM and alleviation of pain on those who have taken remote acupuncture. A randomized 
controlled trial was conducted to find the efficacy of sharphook acupuncture for 4 weeks. They have concluded that sharp-hook acupuncture helps to relieve the pain and restore the shoulder function for patients with PAS [34]. Similarly, in this study also duration of the intervention was 4 weeks. Another study observed that integrating acupuncture with conservative management shows superior effectiveness when compared to conservative management alone [35].

There were different types of needling techniques used to intervene PAS with various number of sessions and duration; no adverse effect was noticed in the previous studies [36,37]. However, in this study both remote acupuncture points (ST38, GB34, GB41, and BL11) and local acupuncture points (LI15, TE14, SI9, and GB21) shows changes within the groups, but when compared with each group remote acupuncture points shows significant changes in the management of periarthritis of shoulder.

\section{Limitations}

Sample size was relatively smaller, no follow-up, room temperature was not evenly maintained to all subjects during treatment, diurnal variations might have influenced the results, other physical activities and diet in home might be acted as confounding factors, mechanism of remote acupuncture points in the management of pain, disability and ROM of shoulder still inconclusive.

\section{Directions for future research}

Replicate with larger sample size with multi-arm randomized controlled trial could be helpful to draw definite conclusion. Strong methodology with follow-up is essential to support our result. Objective variables like digital goniometer \& bio-markers for the pain can be used to have better insight on mechanism of remote acupuncture points.

\section{CONCLUSIONS}

The present study shows that both remote and local acupuncture points may have beneficial effect in the management of shoulder pain and its restriction which was measured through SPADI and range of motion - flexion, abduction and external rotation. Remote acupuncture points showed higher therapeutic benefits on patients with PAS, when compared to local acupuncture points.

\section{CONFLICT OF INTEREST}

The authors declare no conflict of interest.

\section{ORCID}

Kumaresan Poorna Chandran,

Prabu Poorna Chandran, https://orcid.org/0000-0001-9597-557X

Naveena Arumugam, https://orcid.org/0000-0003-1901-1239

Sendhilkumar Muthappan, https://orcid.org/0000-0002-0168-9298 https://orcid.org/0000-0002-8902-0889

\section{REFERENCES}

1. Buchbinder R, Green S. Effect of arthrographic shoulder joint distension with saline and corticosteroid for adhesive capsulitis. Br J Sports Med 2004;38:384-5.

2. Neviaser JS. Adhesive capsulitis of the shoulder: a study of the pathological findings in periarthritis of the shoulder. J Bone Joint Surg 1945;27:211-22.

3. Codman EA. The Shoulder; Rupture of the Supraspinatus Tendon and Other Lesions in or about the Subacromial Bursa. Boston: T. Todd Company, printers, 1934.

4. Ombregt L, Bisschop P, Ter Veer HJ. A System of Orthopaedic Medicine, 2nd ed. London: Churchill Livingstone, 2003.

5. Siegel LB, Cohen NJ, Gall EP. Adhesive capsulitis: a sticky issue. Am Fam Physician 1999;59:1843-52.

6. Manske RC, Prohaska D. Diagnosis and management of adhesive capsulitis. Curr Rev Musculoskelet Med 2008;1:180-9.

7. Morén-Hybbinette I, Moritz U, Scherstén B. The clinical picture of the painful diabetic shoulder-natural history, social consequences and analysis of concomitant hand syndrome. Acta Med Scand 1987;221:73-82.

8. Mangine RE, Heckmann T, Eifert-Mangine M. Alternative techniques for the motion-restricted shoulder. In: Andrews JR, Wilk KE, eds. The Athlete's Shoulder. New York: Churchill Livingstone, 1994:685-94.

9. Jones A, Regan M, Ledingham J, Pattrick M, Manhire A, Doherty M. Importance of placement of intra-articular steroid injections. BMJ 1993;307:1329-30.

10. Rizk TE, Gavant ML, Pinals RS. Treatment of adhesive capsulitis (frozen shoulder) with arthrographic capsular distension and rupture. Arch Phys Med Rehabil 1994;75:803-7.

11. Sun KO, Chan KC, Lo SL, Fong DY. Acupuncture for frozen shoulder. Hong Kong Med J 2001;7:381-91.

12. Dodenhoff RM, Levy O, Wilson A, Copeland SA. Manipulation under anesthesia for primary frozen shoulder: effect on early recovery and return to activity. J Shoulder Elbow Surg 2000;9: 23-6.

13. Andersen NH, Johannsen HV, Sneppen O, Søjbjerg JO. [Frozen shoulder. Arthroscopy and manipulation in general anesthesia, followed by early passive mobilization]. Ugeskr Laeger 1996;158: 
147-50. Danish.

14. Buchbinder R, Youd JM, Green S, Stein A, Forbes A, Harris A, et al. Efficacy and cost-effectiveness of physiotherapy following glenohumeral joint distension for adhesive capsulitis: a randomized trial. Arthritis Rheum 2007;57:1027-37.

15. Bulgen DY, Binder AI, Hazleman BL, Dutton J, Roberts S. Frozen shoulder: prospective clinical study with an evaluation of three treatment regimens. Ann Rheum Dis 1984;43:353-60.

16. Schoen AM. Acupuncture for musculoskeletal disorders. Probl Vet Med 1992;4:88-97.

17. Park HJ, Lim S, Joo WS, Yin CS, Lee HS, Lee HJ, et al. Acupuncture prevents 6-hydroxydopamine-induced neuronal death in the nigrostriatal dopaminergic system in the rat Parkinson's disease model. Exp Neurol 2003;180:93-8.

18. Diehl DL, Kaplan G, Coulter I, Glik D, Hurwitz EL. Use of acupuncture by American physicians. J Altern Complement Med 1997;3:119-26.

19. Vickers AJ, Linde K. Acupuncture for chronic pain. JAMA 2014;311:955-6.

20. Green S, Buchbinder R, Hetrick S. Acupuncture for shoulder pain. Cochrane Database Syst Rev 2005;(2):CD005319.

21. Agrawal AL, Sharma GN. Advanced Acupuncture Therapy. New Delhi: CBS Publishers and Distributors, 2009.

22. Williams JW Jr, Holleman DR Jr, Simel DL. Measuring shoulder function with the Shoulder Pain and Disability Index. J Rheumatol 1995;22:727-32.

23. Roach KE, Budiman-Mak E, Songsiridej N, Lertratanakul Y. Development of a shoulder pain and disability index. Arthritis Care Res 1991;4:143-9.

24. Lee SH, Yoon C, Chung SG, Kim HC, Kwak Y, Park HW, et al. Measurement of shoulder range of motion in patients with adhesive capsulitis using a Kinect. PLoS One 2015;10:e0129398.

25. Mullaney MJ, McHugh MP, Johnson CP, Tyler TF. Reliability of shoulder range of motion comparing a goniometer to a digital level. Physiother Theory Pract 2010;26:327-33.

26. Vanderploeg K, Yi X. Acupuncture in modern society. J Acupunct Meridian Stud 2009;2:26-33.

27. Chen L. [Comparison of therapeutic effects between normal acupuncture and shallow needling with short-needle on periarthritis of shoulder]. Zhongguo Zhen Jiu 2006;26:647-8. Chinese.

28. Chen SJ, Li H, Zhang JW. [Control study of therapeutic effects of three phase acupuncture method and routine acupuncture method on periarthritis of shoulder]. Zhongguo Zhen Jiu 2006; 26:421-3. Chinese.

29. Lu J, Sun JH, Fu ZH, Yuan JH, Li J, Ji AQ. [Transient therapeutic effect and safety of superficial needling therapy for treatment of periarthritis of shoulder]. Zhongguo Zhen Jiu 2008;28:414-6. Chinese.

30. Lathia AT, Jung SM, Chen LX. Efficacy of acupuncture as a treatment for chronic shoulder pain. J Altern Complement Med 2009;15:613-8.

31. Molsberger AF, Schneider T, Gotthardt H, Drabik A. German Randomized Acupuncture Trial for chronic shoulder pain (GRASP) - a pragmatic, controlled, patient-blinded, multi-centre trial in an outpatient care environment. Pain 2010;151:146-54.

32. Han ZX, Qi LL, Chu LX, Cai WQ, Chen XF, Huang JY, et al. [Program optimization in the stage treatment of periarthritis of shoulder with acupuncture, moxibustion and positive functional exercise]. Zhongguo Zhen Jiu 2014;34:1067-72. Chinese.

33. Yang R, Liu YX. [Clinical observation on shoulder periarthritis treated with Bo's abdominal acupuncture in stages]. Zhongguo Zhen Jiu 2012;32:26-30. Chinese.

34. Fu QN, Shi GX, Li QQ, He T, Liu BZ, Sun SF, et al. Acupuncture at local and distal points for chronic shoulder pain: study protocol for a randomized controlled trial. Trials 2014;15:130.

35. Schröder S, Meyer-Hamme G, Friedemann T, Kirch S, Hauck $\mathrm{M}$, Plaetke $\mathrm{R}$, et al. Immediate pain relief in adhesive capsulitis by acupuncture-a randomized controlled double-blinded study. Pain Med 2017;18:2235-47.

36. Zhang JC, Liu S, Lü JL, Chen ZX, Li J. [Periarthritis of shoulder treated with deep puncture by elongated needle combined with spike-hooked needle and cupping: a multicentral randomized controlled study]. Zhongguo Zhen Jiu 2011;31:869-73. Chinese.

37. Ji L, Wang H, Cao Y, Yan P, Jin X, Nie P, et al. Sharp-hook acupuncture (Feng Gou Zhen) for patients with periarthritis of shoulder: a randomized controlled trial. Evid Based Complement Alternat Med 2015;2015:312309. 